\title{
Refresh cell culture
}

Biomedical research needs upgraded standards for the monitoring, control and reporting of the environmental conditions of cells in culture.

W hen working with cells, tissues, organs or individuals, biological heterogeneity and variability are commonplace. However, for some types of heterogeneity and variability, their influence can be reduced or controlled for. Participants in trials are selected according to pre-specified criteria approved by scientific review and ethics committees; cell identity and cell phenotypes can be measured, and the cells sorted according to specific biomarkers; and animals for research and donors of biospecimens can be selected to establish genetically homogeneous or environmentally similar backgrounds. And many standard operating procedures for the processing, maintenance and handling of biospecimens and animals are designed to minimize methodological variabilities.

But, even for routine biomedical work, in particular for cell culture and biospecimen preservation, efforts to reduce environmental or methodological variabilities are often insufficient, and whether these variabilities could have any effects on the experiments is commonly discounted or ignored. As reported by Carlos Duarte, Mo Li, Juan Carlos Izpisua Belmonte and colleagues in a Comment in this issue of Nature Biomedical Engineering, the environmental conditions used in mammalian cell culture are routinely underreported in biomedical studies (the research Articles included in this issue are no exception). Specifically, regardless of the cells used (human or non-human cell lines, primary cells or stem cells, for instance), the $\mathrm{pH}$ of the media and the level of atmospheric oxygen in the culture chamber are seldom specified in the literature, and only about $40 \%$ of biomedical studies report the level of carbon dioxide and the temperature in the culture chamber. Yet it has long been known that the levels of oxygen and carbon dioxide in the environment of cells alter their physiology - in fact, the 2019 Nobel Prize in Physiology or Medicine recognized ${ }^{1}$ work that unveiled how cells sense and adapt to the availability of oxygen. Moreover, about $35 \%$ of biomedical studies do not describe the type of culture system employed (batch culture is usually assumed). Duarte and colleagues derived these statistics by looking at 1,749 cell-culture experiments reported

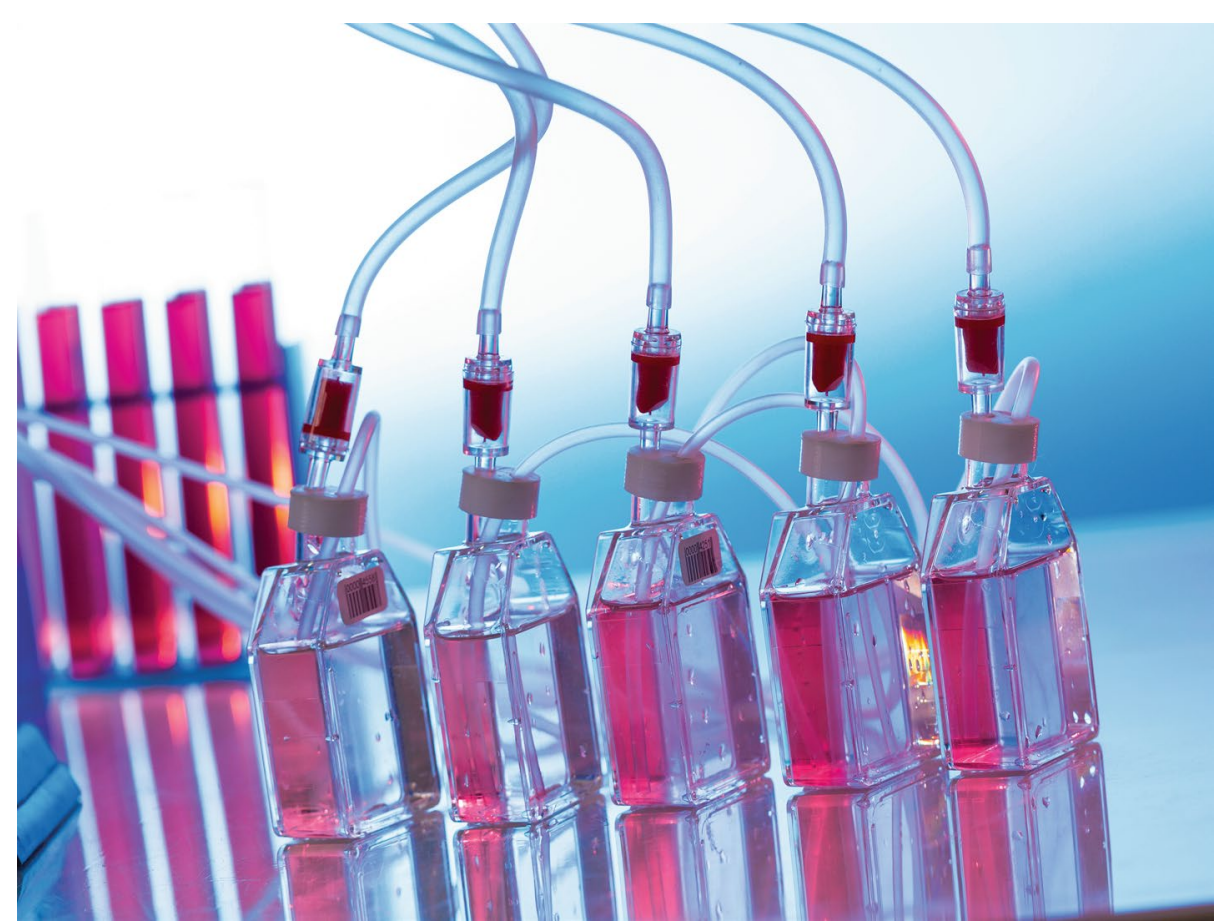

Credit: Wladimir Bulgar / Science Photo Library / Getty

in 688 randomly selected biomedical studies published in 2014-2019.

Why is it important to thoroughly report the environmental conditions, rather than, for example, to assume that there is no need to do so because commercial media and standard nominal set points for temperature and carbon dioxide levels are used? First, not reporting the actual set points may suggest that it was not verified whether the culture conditions were maintained at the set points. Second, such underreporting may falsely imply that any variabilities in the cell-culture conditions were largely irrelevant to the results of the experiments, which may not be the case. In fact, standard batch cultures can undergo environmental drift $^{2}$ (even with daily exchanges of medium), with subsequent alteration of cell growth and cell function; the use of suitable media does not necessarily ensure the stability of the environmental conditions; and, for many types of human cells, physiological function is only retained within the narrow physiological $\mathrm{pH}$ range of 7.2-7.4. Moreover, for biospecimens used after cryopreservation, cryoinjuries - that is, damage to the preserved cells caused by the formation of ice and by stresses caused by cold-storage, freezing or vitrification processes, or by the toxicity of the cryoprotectants used - can affect cell viability, proliferation and function, as highlighted in a Review Article in this issue by Haishui Huang, Xiaoming He and Martin Yarmush. Third, underreporting makes replicability and reproducibility ${ }^{3}$ attempts unnecessarily harder and, for translational research, this may hamper clinical prospects ${ }^{4}$.

The prevalent underreporting of the environmental conditions of cell culture could be partly addressed if journals were to specifically request that authors declare the environmental parameters, the culture set-up, and culturing conditions and procedures (such as cell density, media-exchange rate, rate of sub-culturing, and passage number), and any monitoring and control processes that they followed. Nature Biomedical Engineering will do so as a matter of course. Yet aiming only at the 
underreporting problem will be inadequate. The elephant in the room is the fact that the most relevant environmental conditions of cell culture $-\mathrm{pH}$, and the levels of dissolved oxygen and carbon dioxide - are rarely tracked let alone controlled, as Duarte and colleagues conclude from their analysis of the literature. Less than $1 \%$ of cell-culture experiments in biomedical studies involve bioreactors or chemostats - that is, bioreactors that allow for the continuous addition of fresh medium and the removal of culture liquid - as their acquisition, and maintenance costs are higher than for batch culture. Hence, it is not surprising that periodic measurements of the actual culture conditions (most relevant for cultures that need to be maintained for longer than a few days), which bioreactors more easily allow, are rarely carried out. Setting nominal set points for temperature and the levels of oxygen and carbon dioxide, and assuming that the $\mathrm{pH}$ will be within the physiological range, may be just fine for many studies, particularly with widely used cell lines and for short-term culture, if the cell-culture data are only ancillary to the conclusions, and if the study is well-powered or includes a sufficiently large number of replicates to show the uncertainty levels of the measurements. Yet when using rarer or more delicate biospecimens, particularly primary cells, stem cells, or reproductive cells or tissue (such as sperm cells, oocytes and embryos), verifying - and, when needed, controlling - relevant environmental parameters may be crucial for the interpretation of the findings and for reproducibility.

All in all, monitoring and controlling for the most relevant cell-culture parameters will require awareness of best practices, the development and wide implementation of more stringent yet workable standards, funding for the grunt work needed to better determine the impacts of environmental instabilities on cellular homeostasis, cell function and cell growth, and incentives and penalties ${ }^{5}$ conducive to better reporting -in essence, a refresh of the research culture around cell culture.

Published online: 23 August 2021

https://doi.org/10.1038/s41551-021-00790-1

References

1. Nat. Biomed. Eng. 3, 843-844 (2019).

2. Eagle, H. Science 174, 500-503 (1971).

3. Nat. Biomed. Eng. 2, 789-790 (2018).

4. Ioannidis, J. P. A., Kim, B. Y. S. \& Trounson, A. Nat. Biomed. Eng. 2, 797-809 (2018)

5. Nat. Biomed. Eng. 4, 845-846 (2020). 